\title{
THE INITIAL MASS FUNCTION OF MASSIVE STARS IN GALAXIES: EMPIRICAL EVIDENCE
}

\author{
John M. Scalo \\ Department of Astronomy \\ University of Texas
}

\begin{abstract}
Observational constraints on the form of the high-mass stellar IMF are reviewed. The evidence includes star counts in the solar neighborhood, individual and composite star clusters, and nearby galaxies, and arguments based on integrated light and chemical evolution modeling. There is no convincing evidence for any systematic variations of the shape of the high-mass IMF. However, the various determinations are very uncertain, and do not allow any firm estimate of the logarithmic slope of the upper IMF; the appropriate value is somewhere between -1.3 and -2.3 , with region-to-region variations smaller than about \pm 0.5 . A number of lines of evidence suggest that the lower mass limit or mode mass of the IMF increases with increasing star formation rate, reaching perhaps $10-15 \mathrm{~m}_{\odot}$ in some starburst galaxies. It is also possible that the upper mass limit depends on metallicity, based on variations in excitation conditions of $\mathrm{H}$ II regions.
\end{abstract}

\section{Introduction}

The frequency distribution of stellar masses at birth, called the "initial mass function" (IMF) connects phenomena on the scale of stars with those on the galactic scale, and enters into nearly all studies of galactic evolution. The IMF of massive stars is especially important, since it is these stars that contribute most of the luminosity, ionization, and kinetic energy of star-forming regions in galaxies. Unfortunately, despite many independent studies using a variety of methods, our knowledge of the shape of the highmass IMF remains very uncertain for a number of reasons, although substantial progress has been made in the past few years. The present paper is an attempt to briefly summarize the results and problems associated with a variety of approaches to the determination. A detailed review of this subject, as well as of the IMF of lower-mass stars, is given elsewhere (Scalo 1985).

For consistency's sake, we define the IMF as the number of stars formed at the same time in some volume of space per unit logarithmic mass interval; e.g. the classical Salpeter IMF would have a logarithmic slope of $\Gamma=-1.3$. It is useful to think of the IMF as a probability distribution. This definition assumes that the mechanisms responsible for the form of the IMF constitute a process that is statistically homogeneous in space and stationary in time. We also introduce the parameters $m_{1}$ and $m_{u}$ as the lower and upper mass limits of the IMF. 


\section{Star Counts in the Solar Neighborhood}

The relative number of stars presently observed in each mass interval does not directly give the IMF because of the effects of stellar evolution; stellar lifetimes decrease with increasing mass, so the actual IMF is flatter than the presently observed mass distribution. For high mass stars the conversion does not depend on the history of the star formation rate (SFR) because their lifetimes are small compared to the timescale over which the global SFR has varied. Therefore the IMF can be constructed by dividing the presentlyobserved number of stars in each mass interval by the average stellar lifetime for that mass interval. In our galaxy this star counting technique can sample massive stars out to 1-3 $\mathrm{kpc}$ from the sun.

A number of recent papers have addressed this problem (Lequeux 1979; Garmany et al. 1982, GCC; Bisiacchi et al. 1983, BFS; Humphreys and McElroy 1984, HM; Van Buren 1984, Vanbeveren 1984; Scalo 1985), with differing results. Since masses cannot be directly observed, the present-day mass distribution must be derived either from the distribution of stars in the H-R diagram using theoretical evolutionary tracks and semiempirical effective temperature and bolometric correction calibrations, or from the luminosity function (LF), using a mass-luminosity relation. All the studies listed above except that of Scalo (1985) use some variant of the former method. A major problem with all these studies is the question of completeness.

TABLE 1

\section{Estimates of $\Gamma$ for High-Mass Stars}

$\begin{array}{lcc}\text { Study } & \Gamma & \text { Notes } \\ \text { Lequeux } & -1.3 & \\ \text { GCC } & -1.4 \text { to }-1.7 & \text { a,b } \\ \text { BSF } & -2.0 & \\ \text { HM } & -2.2 & \mathrm{c} \\ \text { VanBuren } & -1.3 & \\ \text { Vanbeveren } & -2.4(-1.9) & \mathrm{d} \\ \text { MMF(LF) } & -1.5 & \mathrm{e}\end{array}$

aRange for 3 different sets of evolutionary tracks.

bDoes not include B stars.

cCorrected for incompleteness in the $15-30 \mathrm{~m}_{\odot}$ bin.

${ }^{d}$ For combined cluster and field star sample; value in parentheses was derived from Vanbeveren's results without weighting.

${ }^{e}$ From $L F$ of GCC for $m>25$, joined to adopted LF at smaller masses.

The results of the method which counts stars in various mass ranges in the H-R diagram are sensitive to the adopted evolutionary tracks and effective temperture scale. The evolutionary tracks present a particularly vexing problem since the quantitative effects and rates of mass loss, nonlocal convection, and internal rotational mixing are not wellunderstood. The same problems occur in using the mass-luminosity relation in 
conjunction with the luminosity function, although the effects are not as severe. On the other hand, the method which counts stars in the H-R diagram is in principle capable of higher accuracy in estimating an IMF slope because the steepness of the mass-luminosity relation means that a given uncertainty in the slope of the luminosity function translates into a much larger uncertainty in the IMF slope.

Another uncertainty, not very well recognized, enters when we divide the star counts by the stellar lifetimes. Main sequence lifetimes are uncertain by perhaps $30-40$ percent, primarily due to our uncertainties concerning the importance of convective overshoot in the core and internal mixing due to rotational or other instabilities. Both of these effects increase the stellar lifetime.

Table 1 summarizes approximate logarithmic slopes of the high-mass $\mathrm{IMF}(\mathrm{m} \geqslant 10$ - $25 \mathrm{~m}_{\odot}$ ) according to a number of investigations. The slope given for Lequeux (1979) assumes that none of the observed stars are stars from an evolved population. The slope given for HM does not include the data point at the highest mass, since the number of stars is small and the appropriate mass is uncertain. The slope given for Van Buren (1984) has been modified to account for the estimated effects of convective overshoot on the luminosities and lifetimes. The result derived from the luminosity function [denoted IMF(LF)] in Table 1 is based on the luminosity function of stars in the GCC catalogue, but joined to other luminosity function determinations at smaller luminosities in order to account for incompleteness at small luminosities. Notice that IMF(LF) is in reasonable agreement with the GCC estimates, suggesting that the two approaches are consistent.

A detailed discussion of all the causes of agreement and disagreement between the various determinations (lifetimes, evolutionary tracks, effective temperature scales, etc.) is beyond the scope of this review; such a discussion can be found in Scalo (1985). The major consideration worth mentioning is that the GCC catalogue included only $\mathrm{O}$ stars. The work of BSF, HM, and Vanbeveren (1984) clearly shows how the inclusion of later spectral types steepens the derived IMF, and, on this basis alone, we might conclude that the IMF slope of massive stars is probably around -2. However, Van Buren (1984) also included later spectral types, a large sample, and a very careful study of incompleteness corrections and reddening, and obtained a slope of -1.3 (or even flatter) for high-mass stars. I have been unable to resolve this discrepancy, and conclude that, without a more careful study and improvements in our knowledge of stellar evolution and the effective temperature scale, the existing studies provide little reason for choosing a particular value of the high-mass IMF slope. This is a disappointing but, I think, realistic conclusion.

\section{Star Counts in OB Associations and Open Clusters}

Additional information on the slope of the high-mass IMF comes from studies of individual (and composite) OB associations and young stellar clusters. Such studies are free from some of the problems mentioned above, but suffer from a number of new difficulties, such as mass segregation, small number statistics, and non-coeval star formation.

A number of papers have attempted to reduce the small-number fluctuations by combining the star counts for a fairly large number of clusters to obtain an average, or "composite" cluster IMF. The construction of such a composite function involves a number of difficulties which cannot be reviewed here. The only work which has attempted to address some of these problems is Taff (1974; see also Vanbeveren 1982).

The composite cluster IMFs derived from composite luminosity functions compiled by Taff (1974) and Burki (1977; large, medium and small diameter clusters shown separately) are shown in Figure 1 and compared with the IMF(LF) derived in 
Scalo (1985). The normalization is arbitrary. For Taff's IMF, the IMF slope for $1<\mathrm{m}<$ 10 is about -1.8. The IMF appears to flatten for $m ? 10$, but the number of stars used is small. Burki's slopes between $\mathrm{m}=7.5$ and $25 \mathrm{~m}_{\odot}$ (small clusters) or $50 \mathrm{~m}_{\odot}$ (large clusters) are -1.7 (small), -1.5 (medium) and -1.2 (large). Perhaps the most important point is that these slopes are flatter than most of the studies of field stars using the H-R diagram reviewed earlier.

Studies of individual young clusters are problematic because of small number statistics and other difficulties, but a comparison of a number of careful studies (Scalo 1985, Sagar et al. 1985) suggests general agreement among themselves and with the composite functions. The IMF slope between about 3 and $10 \mathrm{~m}_{\odot}$ appears to be around 1.5 , but there is strong evidence for flattening at larger masses, especially in M17, NGC 654, and NGC 6611, in agreement with the effect noted above for Taff's (1974) composite cluster IMF.

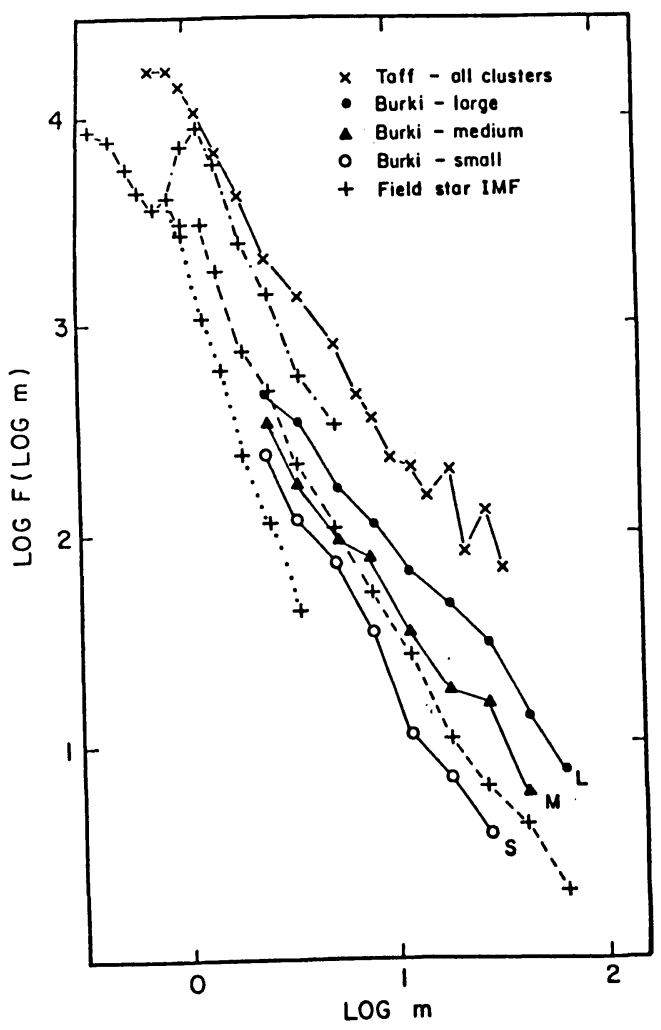

Figure 1. Estimates of composite open cluster IMFs by Taff (1974) and Burki (1977); the field star IMF constructed by Scalo (1985) is also shown. Normalization is arbitrary. 
IMF variations between clusters are notoriously difficult to establish, so I will not attempt any review here, except to note the turnover in the NGC $3293 \mathrm{IMF}$ at around $3 \mathrm{~m}_{\odot}$ found by Herbst and Miller (1983) and the enormous variations in IMF slope found in the maximum likelihood fits of Tarrab (1982).

\section{Star Counts in Nearby Galaxies}

The shape of the IMF and its possible variations on scales $? 1 \mathrm{kpc}$ can be studied using star counts within and among nearby galaxies. After corrections for incompleteness, crowding, foreground stars, etc. (these are nontrivial problems), a luminosity function can be constructed. Comparisons of luminosity functions should give some idea as to whether galactic-scale IMF variations exist. (IMF studies based on counting stars in the $\mathrm{H}-\mathrm{R}$ diagram will not be reviewed here; in my opinion they are of little use for extragalactic systems because of the bright limiting magnitude imposed by the necessity of obtaining spectra).

Several recent comparisons of this nature have been carried out. Scalo (1985) compared the LFs of six galaxies based on an inhomogeneous set of data culled from the literature, including both spirals (M33, M31) and irregulars (LMC, SMC, IC 1613, NGC 6822). Considering the variety of data sources, the agreement was remarkably good. Hoessel and coworkers (see Hoessel, this volume) have derived a homogeneous set of LFs for a number of dwarf irregulars; again, the agreement at the bright end, where incompleteness problems are minimized, is surprisingly good.

The most detailed study of galactic LFs is due to Freedman (see Freedman 1985, and this volume). Her study includes homogeneous CCD data for seven spiral and irregular galaxies, supplemented with data from the literature for three additional irregulars. The largest sample is for M33, with over 5000 stars. Freedman's papers should be consulted for details concerning automated star counting and image identification, crowding corrections, etc. The resulting LFs exhibit, again, a remarkable similarity with a mean slope dlog $\mathrm{N} / \mathrm{dM}_{\mathrm{v}}=0.67 \pm 0.03$. If the LF slope differences estimated by Freedman are taken literally, they imply a variation in the IMF slope of less than \pm 0.5 , and probably much less, considering the uncertainties in the data. Furthermore, Freedman finds no significant variations in slope with position or metallicity in the galaxies studied, a conclusion in agreement with studies of the LMC LF at lower masses (see Scalo 1985).

One must conclude from these three comparisions that there is ne evidence for high-mass IMF slope variations within or among nearby galaxies. This conclusion is especially striking because the galaxies vary greatly in morphology and metallicity.

\section{Indirect Evidence: Integrated Light}

I use the term "indirect" to refer to methods for estimating the IMF that do not employ star counts. The observable quantities used in such approaches include broad-band colors, mass-to-light ratios, spectral line strengths, and elemental abundances and abundance ratios. Most of these methods are very uncertain and ambiguous, but they can sample distant volumes of space and large numbers of objects which cannot be studied by star counts, and, taken together, can yield some clues to the gross properties of the IMF and especially its possible variations in space and time. In the following summary of some of the more recent work in this area, the reader should bear in mind the large uncertainties and ambiguities that accompany all such studies. The following discussion only includes 
methods which give information on the high-mass IMF, and even then the choice of topics is selective. A more comprehensive review is given elsewhere (Scalo 1985).

\section{(a) Mass-to-Light Ratios}

This ratio is roughly a measure of the ratio of the number of low-mass stars (and remnants and other dark matter), which contributed most of the mass, to the number of higher-mass stars, which contribute most of the light (see Faber and Gallagher 1979 for a review). Thus, $M / L$ should be larger for a steeper IMF. In connection with the $\mathrm{IMF}$, the $\mathrm{M} / \mathrm{L}$ ratio has been used mostly as a probe in elliptical galaxies. Recently Terlevich and Melnick (1984) have interpreted an apparent correlation of metallicity $\mathrm{Z}$ with $\mathrm{M} / \mathrm{L}$ in giant $\mathrm{H}$ II regions in galaxies and isolated intergalactic H II regions (as well as elliptical galaxies and globular clusters) in terms of an IMF slope which steepens with decreasing metallicity. They suggest the relation $\Gamma=-4.0-\log Z$ for a pure power-law IMF. A problem is that their $\mathrm{M} / \mathrm{L}$ values for $\mathrm{H} I I$ regions are based on virial masses derived from emission line widths, and these masses may be inappropriate, as discussed by several authors. In addition, the suggested relation does not agree with results from star counts, at least for high-mass stars. Still, this evidence, along with additional correlation studies for elliptical galaxies, suggests at least that the ratio of high-to-low-mass stars may depend on metallicity, but the variation may not involve the slope of the high-mass IMF.

\section{(b) Colors}

Color variations among galaxies cannot in themselves be used to infer IMF variations because broad-band colors are very sensitive to the SFR and other parameters. The best illustration of this fact is provided in the figure presented by Larson and Tinsley (1978) and reproduced in Tinsley (1980) and Scalo (1985). The observed colors of galaxies are certainly consistent with a universal $\mathrm{MMF}$, but they do not allow an estimate of the slope $\Gamma$ and/or the upper mass limit $\mathrm{m}_{\mathrm{u}}$.

\section{(c) Population Synthesis}

Attempts to match observed galaxy colors, spectra, or line strengths by finding a "best" mixture of stars at various spectral types and luminosity classes is beset by problems concerning solution uniqueness and the choice of appropriate constraints, but, in principle, this method could yield an IMF estimate. A good example is provided by the paper of Ellis et al. (1982) for four spiral galaxies.

\section{(d) Ultraviolet Luminosities}

The UV flux spectrum from a young population depends on the IMF and SFR of massive stars. Use of the shape of the spectrum does not appear viable for IMF studies, but attempts to match the absolute UV luminosity are more promising. Donas and Deharveng (1984) have studied the UV fluxes of 40 spiral and irregular galaxies, and suggest $\Gamma=-2$ \pm 0.5 with the estimated limit on the dispersion based on the modest scatter in the derived SFR-gas mass correlation.

(e) Lyman Continuum Indicators 
Probably the best indirect methods for studying the high mass IMF in galaxies involve quantities which allow an estimate of the rate at which photons are emitted by stars in the Lyman continuum, $\mathrm{N}(\mathrm{Lyc})$, since the Lyman continuum flux is dominated by stars with $\mathrm{m}$ $\succsim 20 \mathrm{~m}_{\odot}$. The methods available include hydrogen line luminosities, which are proportional to $\mathrm{N}(\mathrm{Lyc})$, the thermal radio continuum emission from $\mathrm{H} I \mathrm{I}$ regions, and line intensity ratios like $[\mathrm{O} \mathrm{III}] / \mathrm{H} \beta$, which give an estimate of an equivalent temperature usually denoted "T $\mathrm{eff}_{\text {". }}$ All these approaches suffer from severe uncertainties, e.g. the effects of dust, contamination by nonthermal radio emission, sensitivity to model atmospheres, etc. These difficulties are reviewed in Scalo (1985); here we will only summarize a few of the most interesting results, which should be regarded with caution.

(f) The W(H $\alpha)-(B-V)$ Relation for Late-Type Spirals

The ratio of $\mathrm{H} \alpha$ or $\mathrm{H} \beta$ flux to the continuum flux just outside the line is a useful tool for studying IMFs and SFRs in galaxies. These ratios are usually referred to as the H $\alpha$ or $\mathrm{H} \beta$ equivalent widths. The $\mathrm{H} \alpha$ or $\mathrm{H} \beta$ emission is due to massive $\mathrm{O}$ stars (through the Lyman continuum flux), while the continuum radiation comes mostly from B and A stars around $H \beta$ and $1-3 m_{\odot}$ red giants around $H \alpha$. Therefore, these quantities are measures of the ratio of $m \geqslant 20 \mathrm{~m}_{\odot}$ stars to intermediate-mass stars. However, they are also sensitive to the upper mass limit and SFR history.

Kennicutt (1983) has shown how the distribution of galaxies in the W(H $\alpha)-(B-V)$ plane can be used to constrain the SFR histories and IMFs in late-type galaxies by comparing the observed distribution with theoretical galaxy evolution calculations. The comparison is reproduced in Figure 2. Each band corresponds to a different IMF for a range of SFR timescales, with the steepest IMF at the bottom. The best fit is found for the intermediate IMF, which is a power law with $\Gamma=-1.5$. Although the absolute value of this estimate is uncertain (e.g. it depends on the adopted upper mass limit $m_{u}$ if $m_{u} \geqslant 300$ $\mathrm{m}_{\odot}$, and rests on the validity of the theoretical evolution models), the modest spread does suggest that yariations in $\Gamma$ are probably less than about \pm 0.3 , in agreement with the star counts discussed earlier.

A related procedure involving $\mathrm{W}(\mathrm{H} \beta)$-color distributions for clusters in $\mathrm{H}$ II regions has been discussed by DeGioia-Eastwood (1984), and, although there are several problems, the method appears promising for future work.

The weak $\mathrm{H} \alpha$ emission in $\mathrm{Sa}$ and Sab galaxies has been interpreted as due to an IMF deficient in high-mass stars, for example by van den Bergh (1976); but the low excitation could just as well be due to small current SFRs. The question cannot be resolved without very accurate measurements of $\mathrm{H} \alpha$ equivalent widths in Sa and $\mathrm{Sab}$ galaxies. Further discussion of this question as it relates to anemic and smooth-armed spirals and the disk of M82 can be found in van den Bergh (1981) and Scalo (1985).

\section{(g) Starburst Nuclei}

"Starburst nuclei" refers to the central regions of several galaxies which have been found to be extremely luminous and interpreted in terms of very large SFRs, based on 
comparison with theoretical galaxy evolution models. These galaxies are often involved in a galaxy interaction, which is apparently able to trigger the burst by an unknown mechanism. The pioneering study of M82 and NGC 253 by Rieke et al. (1980) was important in establishing the starburst characteristics. More recent studies of IC 2153 by Olafsson et al. (1984), of Mk 171 (= NGC 3690 + IC 694) by Gehrz et al. (1983) and Augarde and Lequeux (1985), and of the extreme starburst galaxies Arp 220 and NGC 6240 by Rieke et al. (1985), as well as other less detailed studies of other galaxies, have for the most part substantiated the conclusions of Rieke et al. (1980) for M82 and NGC 253.

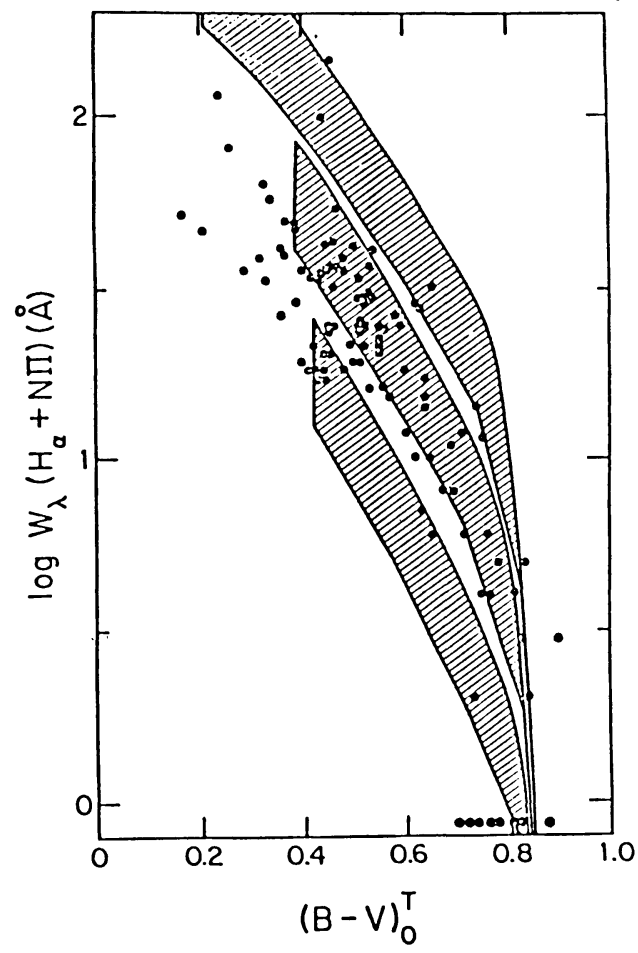

Figure 2. H $\alpha$ equivalent width-(B-V) color diagram for late-type spirals from Kennicutt (1983). Dots are observed galaxies, bands are theoretical calculations for three IMFs with a range of star formation rate histories.

Concerning the $\mathrm{MMF}$, the most striking conclusion is that it is difficult if not impossible to construct acceptable models unless the lower mass limit is very large, from around $3 \mathrm{~m}_{\odot}$ (for M82 and NGC 253) to perhaps $20 \mathrm{~m}_{\odot}$ for Mk 171. Apparently only high-mass stars are formed in these bursts. Although the result can be questioned on several grounds in individual cases (see Scalo 1985), the agreement of these independent studies based on differing constraints lead me to believe that the result is valid. The result is also consistent with other lines of evidence, discussed below, that in regions of large SFRs the lower mass cutoff or the mode (peak) of the IMF may be one to two orders of magnitude larger than in the solar neighborhood. In my opinion, these cases present the only convincing evidence for a significant variation in the large-scale IMF. However, there is as yet no compelling evidence that the shape of the IMF above the cutoff or mode varies. 
(h) Blue Compact Galaxies and Related Objects

Several workers have attempted to infer the SFR histories of the blue compact galaxies, often referred to as extragalactic $\mathrm{H}$ II regions, using galactic spectrophotometric modeling. A secondary goal of these studies has been to provide constraints on the IMF. The IMF of these galaxies is especially interesting because of their large star formation rates and small metallicities. Unfortunately, as in all such investigations, it is extremely difficult to disentangle the history of the SFR from the forn of the IMF, unless several observational constraints are used.

One of the most detailed studies is that of Viallefond and Thuan (1984) on I Zw36. The observational constraints included the $\mathrm{H} \beta$ equivalent width, related to the ratio of $\mathrm{O}$ stars to B and A stars, the "effective temperature" $T_{\text {eff }}$, which depends strongly on the high mass IMF slope $\Gamma$ and the upper mass limit $m_{u}$, and ratios of continuum intensities at (mainly) two pairs of wavelength in the visual part of the spectrum, which are related to the IMF of stars of spectral type A to B2. For the high mass stars, the models require $\Gamma \approx$ -1.5 and $\mathrm{m}_{\mathrm{u}} \approx 100 \mathrm{~m}_{\odot}$. If the SFR has been constant with time, the lower mass limit must be about $4 \mathrm{~m}_{\odot}$ to account for the large value of $\mathrm{W}(\mathrm{H} \beta)$, a result similar to those found for interacting starburst galaxies. This again suggests $m_{1}$ or the IMF mode value increases with SFR. However, if the burst is assumed to be instantaneous at some time in the past, smaller values of $m_{1}$ would be allowed. A comparison for $I Z w 18$ also gives $\Gamma \approx-1.5$ and $\mathrm{m}_{\mathrm{u}} \approx 100 \mathrm{~m}_{\odot}$, but only an upper limit on $\mathrm{m}_{1}$ of $2 \mathrm{~m}_{\odot}$ could be determined.

In contrast, Viallefond and Thuan find that the giant H II region NGC 5471 in the spiral galaxy M101 can be modeled with $\Gamma \approx-2, \mathrm{~m}_{\mathrm{u}} \approx 100 \mathrm{~m}_{0}$, and $\mathrm{m}_{1} \approx 0.007$. They point out that the derived IMF slope is steeper in the more metal-rich region.

A similar metallicity-dependent IMF slope has been suggested for giant $\mathrm{H}$ II regions in nearby galaxies and blue compact galaxies by Terlevich and Melnick (1985) on the basis of correlations between $H \beta$ strength, $M / L_{B}, T_{\text {eff }}$, and $Z$ : As $Z$ decreases, $H \beta$ and $T_{\text {eff }}$ increase while $M / L_{B}$ decreases. But, as mentioned earlier, the correlations, except for the $\mathrm{T}_{\text {eff }} \mathrm{Z}$ relation, are based on assumed virial equilibrium of the regions studied, which has been called into question in several papers. Even if the result is valid, it only implicates a variation in the relative number of high and low mass stars (the IMF was assumed to be a pure power law from $m_{u}$ to $m_{1}$ ), and may not reflect variations in IMF shape for the massive stars. As mentioned earlier, there is no evidence for a Zdependent $\Gamma$ for high mass stars from star counts in nearby galaxies, and the suggested $Z$ dependent $\Gamma$ does not agree with determinations based on star counts of local stars (see Scalo 1985 for a detailed discussion).

The result of Terlevich and Melnick (1984) which does survive all this scrutiny is the anticorrelation between $\mathrm{T}_{\text {eff }}$ and $\mathrm{Z}$, which has been known for some time (e.g. Alloin et al. 1979). However, with this relation alone it is not possible to decide whether the effect is due to a flatter IMF or a larger $m_{u}$ at smaller $Z$. Further confusion arises because the definition of " $\mathrm{T}$ eff" used by Terlevich and Melnick is inappropriate for a comparison with excitation models, and that the calculated $\mathrm{T}_{\text {eff }}$, however it is defined, is very sensitive to the adopted model atmospheres, as pointed out by Lequeux (private communication). Because of the latter complication, as well as the presence of dust, it is possible that a Z- 
dependence of $\mathrm{T}_{\text {eff }}$ might exist which has nothing to do with the IMF. Obviously, the situation remains confused and deserves further study.

\section{Indirect Evidence: Chemical Evolution Models}

Attempts to constrain the IMF using chemical evolution models are very problematical, and so only a brief summary of the relevant arguments will be given here. Besides the uncertainty in elemental yields from massive stars as a function of mass, the conclusions concerning anomalous IMFs are in nearly all cases ambiguous; they cannot be disentangled from uncertainties in the chemical evolution model. There are additional problems concerning observational uncertainties and models for supernovae, which will be mentioned below. These issues are discussed in detail in Tinsley (1980) and Scalo (1985).

A well-known example concerns the metallicity distribution of disk dwarfs. A closed homogeneous chemical evolution model which starts with no metals and evolves with a constant IMF predicts too many low-Z stars. One solution is to postulate an IMF at early times which was enhanced in high-mass stars, using either pre-enrichment of the disk by an earlier generation of massive stars, or a disk IMF enriched in massive stars at early times. The IMF could have had a flatter slope or a larger value of $m_{1}$.

However, another possible resolution of the discrepancy is to relax the assumptions of the model. Examples include early enrichment of the disk by metals expelled from halo stars with a normal IMF (Ostriker and Thuan 1975) or later infall of metal-poor gas (Lynden-Bell 1975, Lacey and Fall 1983), both of which are consistent with Larson's (1976) dynamic models for disk galaxy formation. One could also speculate that the efficiency of star formation decreases strongly with decreasing metallicity (Talbot and Arnett 1973). At present there is no way to decide between these solutions and the assumption of a variable IMF.

Another argument for an early IMF enriched in massive stars, often mentioned in the literature, is that the $\mathrm{O} / \mathrm{Fe}$ ratio is large in metal-poor stars, while $\mathrm{C} / \mathrm{Fe}$ is roughly constant (see Sneden 1985 for a review of the data). Sneden et al. (1979) pointed out that, since the yields predicted by stellar evolution calculations predict that $O / F e$ increases with mass while $\mathrm{C} / \mathrm{Fe}$ is relatively independent of mass, the observations suggest that the halo IMF was greatly enhanced in very massive stars relative to the disk MMF; according to Twarog and Wheeler (1981), the halo IMF would have had to contain most of its mass in stars with $\mathrm{m} ? 50 \mathrm{~m}_{\odot}$.

Although this conclusion may in fact be correct, it is not generally recognized that the argument depends crucially on the assumption that $\mathrm{C}, \mathrm{O}$, and $\mathrm{Fe}$ are produced in shortlived massive stars, an assumption which now appears doubtful. Explosions of accreting white dwarfs, which are currently the leading contenders for Type I supernovae, must eject large quantities of Fe (see Nomoto 1984, Woosley et al . 1984 and references therein), and possibly Si, S, and Ca (Nomoto et al. 1984). This would explain the behavior of the O/Fe ratio and possibly the observed constancy of S/Fe found by Clegg et al. (1983). In addition, single intermediate-mass stars exploding by carbon deflagration can also account for the observed O/Fe-Fe/H relation (Matteucci and Tornambe 1984). However, in order to escape the IMF argument, a long-lived source of $C$ is required. The ability of exploding white dwarfs to produce significant $C$ is uncertain at present. An alternate source of $C$ is carbon stars, most of which have small masses (see Scalo 1981).

Evidently the use of abundance ratios of primary elements to constrain the IMF is not as clear-cut as is often supposed.

The existence of negative radial metallicity gradients in galaxies has also been invoked as an argument for a variable IMF, with a larger proportion of high-mass stars at 
small galactocentric distances, because the simple closed model for chemical evolution cannot account for such gradients. As pointed out below, there is no evidence for such an IMF slope gradient in M33 (Freedman 1985), which has a modest Z-gradient; nor is there any evidence from star counts for a Z-dependent IMF slope among other galaxies. Again, there exist alternative explanations involving modifications of the chemical evolution model. These include a gradient in the metallicity of infalling gas and/or the ratio of SFR to infall rate, both of which effects are found in Larson's (1976) dynamical models, and radial gas flows (Mayor and Vigroux 1981, Lacey and Fall 1985).

Perhaps the most promising explanation for the observed Z-gradients is that proposed by Güsten and Mezger (1983). Their model involves a variation in the IMF, but this variation is not supposed to be caused by Z-variations. Instead, Güsten and Mezger suggest that the IMF has the same shape everywhere, but the lower mass limit is large, $\mathrm{m}_{1}$

2-3 $\mathrm{m}_{\odot}$, for stars formed in spiral arms, and small, $\mathrm{m}_{1} \approx 0.1 \mathrm{~m}_{\odot}$, for stars formed in interarm regions. A Z-gradient arises because the SFR in arms at a given galactocentric distance $\mathbf{R}$ is assumed to be proportional to the rate at which interarm gas encounters arms, which is larger at smaller $R$ because of differential galactic rotation. Therefore, the ratio of arm to interarm SFRs, and hence the ratio of stars with $\mathrm{m} \geqslant 2-3 \mathrm{~m}_{\odot}$ to stars with $\mathrm{m} \geqslant 0.1$ $\mathrm{m}_{\odot}$, increases with $\mathrm{R}$. Besides reducing uncomfortably large SFRs inferred for spiral galaxies, as explained in Güsten and Mezger's paper, the proposal that only high-mass stars form in spiral arms is consistent with analyses of surface photometry in nearby spirals by Jensen et al. (1981) and Bash and Visser (1981), and fits in well with the suggestion made above that $m_{1}$ or the IMF mode increases in regions of large star formation rates.

Space precludes a discussion of the suggestion that the distribution of dwarf irregular and blue compact galaxies in the metallicity-gas fraction plane can be attributed to a dependence of the IMF on Z (see Scalo 1985 for a review). Suffice it to say that, besides the large uncertainties in the empirical gas masses, the observations can also be explained by large infall rates (perhaps implied by the prevalence of large $\mathrm{H}$ I halos in these galaxies), or galactic winds energized by supernova explosions, as shown in the thorough examination of the problem by Matteucci and Chiosi (1983).

In summary, every argument for a variable IMF based on chemical evolution arguments can be countered by a revision in the chemical evolution model or in the assumptions concerning the masses of stars which produce different elements. The observed metallicity distribution of dwarfs and the large $\mathrm{O} / \mathrm{C}$ ratios in halo-population stars both suggest an IMF enriched in massive stars during the collapse of our galaxy, and such a conclusion is consistent with the suggestion already made several times above, that the lower mass limit or mode mass of the IMF increases with increasing SFR, assuming that our galaxy experienced a large global SFR during the protogalactic collapse phase. It should also be pointed out, however, that globular clusters, which presently contain only low-mass stars, should then contain huge numbers of stellar remnants and have very large $\mathrm{M} / \mathrm{L}$ ratios. Larson (1985) has pursued this idea and suggests that these halo remnants (most of which would not presently reside in globular clusters, since most globular clusters are believed to have been disrupted by various processes during our galaxy's evolution) may account for the dark matter inferred to exist in the halo of our own and other galaxies.

\section{Radial IMF Gradients in Galaxies}

The question of systematic variations of the IMF with position in our own and other galaxies and its possible relation to spatial metallicity variations has received recurrent attention over the past ten years. Because the literature in this area is voluminous and the 
results often conflicting and/or ambiguous, only a short list of the major lines of evidence will be given here.

(a) Luminosity Functions

Burki (1977) found a correlation between upper LF slope and diameter for a sample of 27 young clusters, in the sense that the LF was flatter for larger clusters. Burki argued that, because mean cluster size increases with galactocentric distance, this result could be interpreted as a steepening of the IMF toward the galactic center. However, since Burki did not specifically group the clusters according to galactocentric distance, it is difficult to judge the validity of this conclusion. The young cluster IMF slopes estimated by Tarrab (1982) show no evidence for such a correlation (Scalo 1985), in agreement with the conclusion of Sagar et al. (1985) for a smaller number of better-studied clusters.

Garmany et al. (1982) claimed to have found a gradient in the IMF of O stars, with a flatter IMF toward the galactic center (opposite to the sense proposed by Burki). However, the LF of the sample does not show the effect, except for the faintest stars with $M_{v}>-5$, for which the counts appear incomplete (Scalo 1985). Humphreys and McElroy (1984) find no radial gradient in the bolometric LF of a sample which includes supergiants and $B$ stars as well as the $O$ stars, and show that the gradient proposed by Garmany et al. is probably due to a greater degree of incompleteness for stars toward the galactic center.

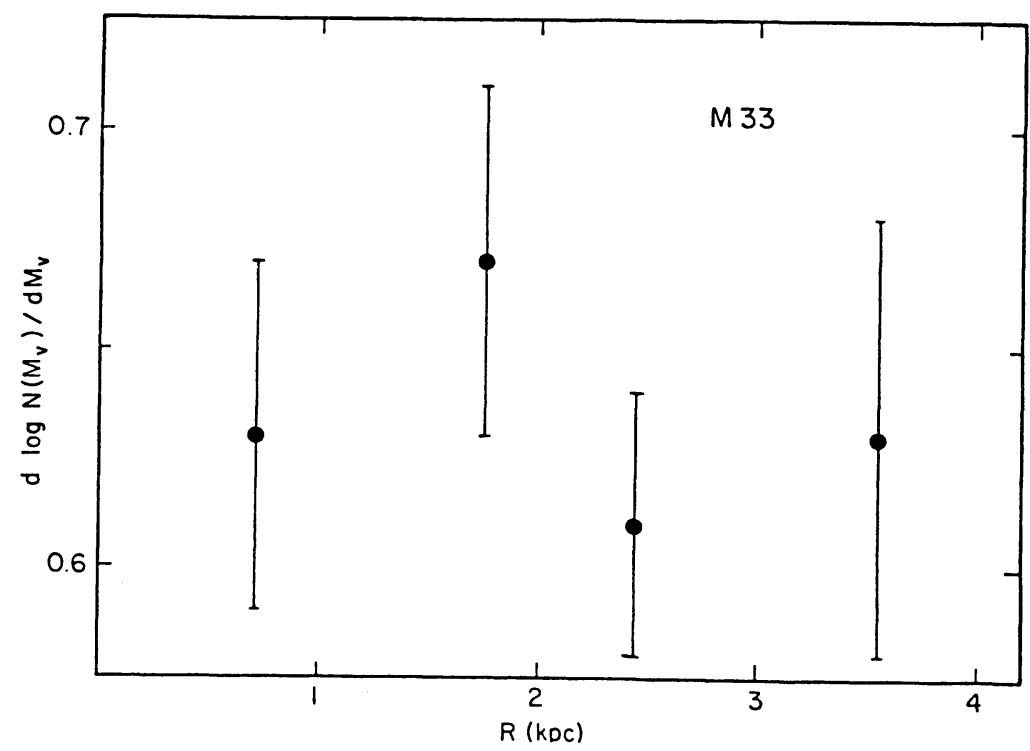

Figure 3. Logarithmic slope of the luminosity function as a function of galactocentric distance in M33, based on the results of Freedman (1985).

The available information on LFs at different positions within individual external galaxies gives no evidence for SMF variations. For the LMC, the studies of several regions outside the bar by Butcher (1977), Stryker and Butcher (1981), and Hardy (1979), 
and the study of a region near the northwest end of the bar by Hardy et al. (1984) all gave LFs in good agreement with each other and with the solar neighborhood function for various $M_{v}$ ranges between -2 and +4 , corresponding to a mass range from $8 m_{\odot}$ to about $1.2 \mathrm{~m}_{\odot}$. Freedman (1985) searched for radial variations in the LFs of several galaxies and found no such variations. The logarithmic slopes of the LFs for 4 difference radial intervals in M33 (the galaxy with the largest sample, over 5000 stars) are shown in Fig. 3. The estimated M33 metallicity gradient implies no dependence of LF shape on metal abundance over a factor of two change in metal abundance. In addition, Freedman found very similar LFs in the northern and southern regions, and in the arm and interarm regions. If the limits on LF slope variations are translated into power law IMF slope variations, the implied variation in the IMF slope is less than about \pm 0.3 .

In summary, there is no convincing evidence for internal IMF variations in our own or other galaxies based on star counts.

\section{(b) Infrared Excesses}

The ratio of infrared flux to Lyman continuum flux, the so-called infrared excess or IRE, is sensitive to the IMF slope and $m_{u}$, but is difficult to interpret because of the unknown fraction of Lyc photons absorbed by dust.

Boisse et al. (1981) and Caux et al. (1985) found a gradient in the IRE of galactic $\mathrm{H}$ II complexes, with larger values of IRE toward the galactic center, although the gradient found by Caux et al. was much smaller than that of Boisse $e t$ al. These results suggest that the IMF is progressively deficient in massive stars toward the galactic center: either a steeper slope or a smaller $\mathrm{m}_{\mathrm{u}}$. However, Scoville et al. (1983) and Lester et al. (1984) have pointed out problems associated with the large beamsize of the IRE measurements which would reduce or eliminate the proposed gradient. Combined with the fact that variations in the IRE can be caused by variations in the dust optical depth and the stellar birthrate, I conclude that IRE variations which could be linked to IMF variations are not well-established. More high-resolution for IR measurements of distant $\mathrm{H}$ II regions are needed to improve the situation.

\section{(c) Radial Excitation Gradients}

Spiral galaxies exhibit radial gradients in excitation conditions as measured by the [O III/H $\beta$ ] ratio, excitation increasing with galactocentric distance. Some of the effect can be attributed to a metallicity gradient, but some of it may reflect a gradient in the effective temperatures of the exciting stars, possibly requiring more massive stars at larger galactocentric distances. Shields and Tinsley (1976) investigated the contribution of a dependence of $\mathrm{m}_{u}$ of $\mathrm{Z}$ (although their abstract states that consistency was found). However, as reviewed in Scalo (1985), even marginal consistency requires an extremely steep IMF with $\Gamma \leqslant-3$, which now appears unreasonable. Because of the sensitivity to various adopted scaling relations and other problems, it appears that the method used by Shields and Tinsley to test for a dependence of $m_{u}$ on $Z$ is currently indeterminate. At any rate, the scaling relations used by Shields and Tinsley and the empirical $\mathrm{W}(\mathrm{H} \beta)$ gradient in M101 are inconsistent with any significant dependence of $m_{u}$ on Z.

Panagia (1980) discussed effects which might account for the systematic decrease in the observed $\mathrm{He}^{+} / \mathrm{H}^{+}$ratios in giant $\mathrm{H}$ II regions toward the galactic center, and concluded that the dominant effect is a dependence of the upper mass limit $m_{u}$ on $Z$, with 
larger $m_{u}$ at smaller $\mathrm{Z}$. I have not attempted to review this argument and its associated uncertainties (e.g. dependence on stellar opacity details and the treatment of dust) in detail.

The matter of a dependence of $\mathrm{m}_{\mathrm{u}}$ on $\mathrm{Z}$ as an explanation of ionization and excitation gradients remains open. An argument against such a Z-dependent $m_{u}$ is that it would lead to an additional systematic effect in the correlation between luminosity of the brightest star in a galaxy and the total galaxy luminosity, an effect which is not seen (see Scalo 1985). On the other hand, there is an apparent anticorrelation between $T_{\text {eff }}$ and $Z$ among galaxies, suggesting an anticorrelation of either $m_{u}$ or the IMF slope with $Z$; in my opinion, this anticorrelation is the only compelling evidence for a possible dependence of $\mathrm{m}_{\mathrm{u}}$ on $\mathrm{Z}$.

\section{(d) Spiral Arm-Interarm Bimodality}

There are three lines of evidence which suggest that stars less massive than about 1 to $3 \mathrm{~m}_{\odot}$ do not form efficiently in spiral arms compared to more massive stars: The detailed analysis of UBVR H $\alpha$ surface photometry of M83 by Jensen et al . (1981); the comparison of arm colors and widths predicted by a dynamical model with observations of M81 by Bash and Visser (1981); and the demonstration by Güsten and Mezger (1983) that the assumption of a lower mass limit $m_{1} \sim 2-3 m_{\odot}$ in the arms and $m_{1} \sim 0.1 m_{\odot}$ for stars formed outside the arms can account for the observed radial oxygen abundance gradient in our galaxy, as well as alleviate the uncomfortably small gas consumption timescales in our own and other spiral galaxies. Details of the arguments and their uncertainties can be found in Scalo (1985). Taken together, these independent studies strongly suggest that the lower mass limit or the mode mass of the IMF is much larger in spiral arms than in interarm regions.

This conclusion is consistent with the studies cited earlier that found evidence for only higher-mass star formation in starburst galaxies. The implication is that the lower mass limit or mode mass of the $\mathrm{IMF}$ increases with increasing star formation rate. Such an IMF is not intrinsically bimodal, but may appear bimodal or multimodal after a given period of time due to the superposition of time-dependent IMFs which arise as the SFR varies. A related interpretation, discussed by Larson (1985), is that the IMF is strictly bimodal and time-dependent. As shown by Larson, this hypothesis can account for a number of observed properties of galaxies. From a theoretical point of view, one idea which can account for the inferred IMF behavior is that discussed by Silk (1977), in which the enhanced cloud heating associated with an increasing number of high-mass stars increases the Jeans mass, and, hence, the characteristic mass. Any such coupling between the radiation field and fragment mass will result in the type of dependence of $m_{1}$ or mode mass on SFR which is suggested by the observations.

\section{Conclusions}

All of the evidence summarized above is consistent with the hypothesis that the form of the massive star IMF, taken over scales $\gtrsim 1 \mathrm{kpc}$, is universal. If variations exist, then, for a power law IMF, the variations in logarithmic slope must be less than \pm 0.5 , and probably smaller. There is no compelling evidence for any systematic slope variations depending on metallicity, galactocentric distance, or galaxy morphology. Unfortunately, the uncertainties are so large that the appropriate value of the slope cannot be reliably estimated; values between -2.4 and -1.3 are possible for $m ? 15 m_{\odot}$. 
On the scales of open clusters, variations in the IMF form do seem likely. In particular, several open cluster IMFs appear much flatter than the field star IMF at large masses. Since most massive stars are probably formed in OB associations, this suggests that open cluster IMFs are flatter than association IMFs at large masses, although this conclusion must still be regarded as tentative.

More convincing arguments for IMF variations concern the upper and lower mass limits. There is some evidence, mainly from systematic variations in excitation conditions in $\mathrm{H} \mathrm{II} \mathrm{regions,} \mathrm{that} \mathrm{m}_{\mathrm{u}}$ increases with decreasing metal abundance, but there is other evidence which conflicts with this idea. Several lines of argument suggest that the lower mass limit or mode mass of the IMF increases strongly with increasing star formation rate. The most extreme examples are certain starburst galaxies in which the lower mass limit or mode mass may be as large as $5-15 \mathrm{~m}_{\odot}$.

\section{References}

Alloin, D., Collin-Souffrin, S., Joly, M., and Vigroux, L. 1979, Astr. Ap., 78, 200.

Augarde, R., and Lequeux, J. 1985, Astr. Ap., in press.

Bash, F. N., and Visser, H. C. D. 1981, Ap. J., 247, 488.

Boisse, P., Gispert, R., Coron, N., Wijnbergen, J. J., Serra, G., Ryter, C., and Puget, J. L. 1981, Astr. Ap., 94, 265.

Burki, G. 1‘977, Astr. Ap., 57, 135.

Butcher, H. 1977, Ap. J., 216, 372.

Caux, E., Puget, J. L., Serra, G., Gispert, R., and Ryter, C. 1985, Astr. Ap., 144, 37.

Clegg, R. E. S., Lambert, D. L., and Tomkin, J. 1983, Ap. J., 250, 262.

De Gioia-Eastwood, K. 1984, P. A. S. P., 96, 625.

Donas, J., and Deharveng, J. M. 1984, preprint.

Ellis, R. S., Gondhalekar, P. M., and Efstathiou, G. 1982, M. N. R. A. S., 210, 223.

Freedman, W. L. 1985, Ap. J., in press.

Garmany, C. D., Conti, P. S., and Chiosi, C. 1982, Ap. J., 263, 777.

Gehrz, R. D., Sramek, R. A., and Weedman, D. W. 1983, Ap. J., 267, 551.

Güsten, R., and Mezger, P. G. 1983, Vistas in Astronomy, 26, 159.

Hardy, E. 1979, A. J., 83, 319.

Hardy, E., Buonanno, R., Corsi, C. E., Jancs, K. A., and Schommer, R. A. 1984, Ap. $J ., 278,592$.

Herbst, W., and Miller, D. P. 1982, A. J., 87, 1478.

Humphreys, R. M., and McElroy, D. B. 1984, Ap. J., 284, 565.

Jensen, E. B., Talbot, R. J., and Dufour, R. J. 1981, Ap. J., 243, 716.

Kennicutt, R. C. 1983, Ap. J., $272,54$.

Lacey, C. G., and Fall, S. M. 1983, M. N., 204, 791.

Lacey, C. G., and Fall, S. M. 1985, Ap. J., 290, 154.

Larson, R. B. 1976, M. N. R. A. S., 176, 31.

Larson, R. B. 1985, preprint.

Larson, R. B., and Tinsley, B. M. 1978, Ap. J., 219, 46.

Lequeux, J. 1979, Astr. Ap., 80, 35.

Lester, D. F., Dinerstein, H. L., Werner, M. W., and Harvey, P. M. 1985, Ap. J., in press.

Lynden-Bell, D. 1975, Vistas in Astronomy, 19, 299.

Matteucci, F., and Tornambé, A. 1984, IAU Symp. No. 105, in Observational Tests of Stellar Evolution Theory, eds. A. Maeder and A. Renzini (Dordrecht: Reidel), 577. 
Mayor, M., and Vigroux, L. 1981, Astr. Ap., 98, 1.

Nomoto, K. 1984, in Stellar Nucleosynthesis, eds. C. Chiosi and A. Renzini (Dordrecht: Reidel), 205, 238.

Nomoto, K., Thielemann, F.-K., and Yokoi, K. 1985, submitted to Ap. J.

Olafsson, K., Bergvall, N., and Ekman, A. 1984, Astr. Ap., 137, 327.

Ostriker, J. P., and Thuan, T. X. 1975, Ap. J., 202, 353.

Panagia, N. 1980, in Radio Recombination Line, ed. P. A. Shaver (Dordrecht: Reidel), 99.

Rieke, G. H., Cutri, R. M., Black, J. H., Kailey, W. F., McAlary, C. W., Lebofsky, M. J., and Elston, R. 1985, Ap. J., 290, 116.

Rieke, G. H., Lebofsky, M. J., Tompson, R. I., Low, F. J., and Tokunaga, A. T. 1980, Ap. J., 238, 24.

Sagar, R., Piskunov, A. E., Myakutin, V. I., and Joshi, V. C. 1985, preprint.

Scalo, J. M. 1981, in Physical Processes in Red Giants, eds. I. Iben and A. Renzini (Dordrecht: Reidel), 77.

Scalo, J. M. 1985, Fund. Cosmic Phys., in press.

Scoville, N. Z., Becklin, E. E., Young, J. S., and Capps, R. N. 1983, Ap. J., 271, 512.

Shields, G. A., and Tinsley, B. M. 1976, Ap. J., 203, 66.

Silk, J. 1977, Ap. J., $214,718$.

Sneden, C. 1985, in ESO Workshop on Production and Distribution of $C, N, O$ Elements, in press.

Sneden, C., Lambert, D. L., and Whitaker, R. W. 1979, Ap. J., 234, 964.

Stryker, L. L, and Butcher, H. R. 1981, in IAU Colloq. No. 68, eds. A. G. Davis Philip and D. S. Hayes (Schenectady: Davis), p. 255.

Taff, L. G. 1974, A. J., 79, 1280.

Talbot, R. J., and Arnett, W. D. 1973, Ap. J., 186, 69.

Tarrab, I. 1982, Astr. Ap., 109, 285.

Terlevich, R., and Melnick, J. 1985, M. N.R.A. S., in press.

Tinsley, B. M. 1980, Fund. Cos. Phys., 5, 287.

Twarog, B. A., and Wheelerf, J. C. 1981, Ap. J., 261, 636.

Vanbeveren, D. 1982, Astr. Ap., 115, 65.

Vanbeveren, D. 1984, Astr. Ap., 139, 545.

Van Buren, D. 1985, Ap. J., in press.

van den Bergh, S. 1976, A. J., 81, 797.

van den Bergh, S. 1981, P. A. S. P., 93, 712.

Viallefond, F., and Thuan, T. X. 1984, Ap. J., 269, 444.

Woosley, S. E., Axelrod, T. S., and Weaver, T. A. 1984, in Stellar Nucleosynthesis, eds. C. Chiosi and A. Renzini (Dordrecht: Reidel).

Discussion : SCALO.

ZINNECKER :

A comment regarding the upper stellar mass limit : it seems to me that on the basis of theoretical considerations collapsing protostars cannot be more massive than 100-150 M. However, one possible way to overcome this limit is the merging of two or several massive stars (see the related poster paper). 